\title{
Incidencia de las pasantías en el perfil del profesional contable
}

\author{
Diana Ramona Ledezma Torres \\ dianitaledezma.dl@gmail.com \\ Facultad de Humanidades y Ciencias de la Educación \\ Universidad Nacional de Pilar
}

\section{RESUMEN}

Las pasantías profesionales son consideradas unas de las estrategias académicas implementadas por las IES para el logro de las capacidades y competencias y con ellas el perfil de egreso de los alumnos, aspecto más que importante para el desempeño del profesional, de allí parte esta investigación bibliográfica que busca describir los diferentes enfoques presentados por teóricos y los resultados de investigaciones sobre la incidencia de las pasantías en el perfil del profesional contable, para el análisis se optó por la investigación bibliográfica, los materiales fueron obtenidos por medio del portal CICCO y de Google Académico. Los principales resultados indican que efectivamente las pasantías inciden en el logro del perfil propuesto por las IES, los referentes de este campo profesional sostienen que estos profesionales deben adquirir conocimientos mediante la experiencia práctica, hacen énfasis en la necesidad asumir un nuevo enfoque de formación para las competencias y que los alumnos se relacionen a las empresas, con los códigos de conducta y funcionamiento que operan en la realidad.

Palabras clave: capacidades; competencias; incidencia, perfil de egreso, profesional contable. 


\title{
Incidence of internships on the profile of the accounting professional
}

\begin{abstract}
Professional internships are considered one of the academic strategies implemented by HEIs for the achievement of skills and competencies and with them the graduation profile of the students, an aspect that is more than important for the performance of the professional, hence this bibliographic research that It seeks to describe the different approaches presented by theorists and the results of research on the incidence of internships on the profile of the accounting professional, for the analysis we opted for bibliographic research, the materials were obtained through the CICCO portal and Google Scholar. The main results indicate that the internships effectively affect the achievement of the profile proposed by the HEIs, the referents of this professional field argue that these professionals must acquire knowledge through practical experience, they emphasize the need to assume a new training approach for the competences and that students relate to companies, with the codes of conduct and operation that operate in reality.
\end{abstract}

Keywords: capacities; incidence, graduation profile, accounting professional.

Artículo recibido: 10. Junio. 2021 Aceptado para publicación: 16. Julio. 2021 Correspondencia: dianitaledezma.dl@gmail.com Conflictos de Interés: Ninguna que declarar 


\section{INTRODUCCIÓN}

Las IES indiscutiblemente representan una de los más importantes medios de vinculación de los profesionales con la sociedad, Castillo, et. al (2013), indican que la vinculación de la universidad con el entorno no es un tema nuevo, continúan su planteamiento indicando que: Se ha ido perfeccionando en función de su misión, en la medida que el desarrollo científico técnico avanza, este problema debe ir transformándose. En la actualidad urge una mayor interacción de manera tal que se favorezcan los procesos de gestión y formación, y que esto a su vez conlleve a un mayor beneficio de la sociedad (p. 4).

Silva Laya (2008) menciona, que si bien la formación para el ejercicio profesional no es la única función que deben cumplir las instituciones de educación superior (IES), es incuestionable que ésta es una de sus funciones vitales. Ante tal importancia, conviene preguntarse qué tipo de formación deberían otorgar, qué competencias son las prioritarias en el contexto socioeconómico y cultural actual y, por supuesto, cuál es la contribución efectiva de las IES al desarrollo de tales competencias (p. 774).

Específicamente el logro de esas competencias contribuirá a obtener el perfil propuesto por las IES, Vega Villacís et al., (2020) indican que las Prácticas y/o Pasantías PreProfesionales son actividades de aprendizaje orientadas a la aplicación de conocimientos, al desarrollo de competencias y habilidades en el campo profesional, que permitan introducir al estudiante al entorno laboral con el apoyo de instituciones o empresas del sector público y/o privado (p.938).

Sobre las pasantías Ibarrola, (1986) destaca que el término de pasantía se utiliza para designar a las actividades realizadas por los pasantes, es decir, los alumnos que ya habían cursado todas las asignaturas correspondientes al plan de estudios pero que aún se encontraban en la realización de trámites para titulación (servicio social, elaboración de tesis, examen profesional, etc.)

Gutiérrez Bernal et al., (2019) mencionan que las IES cumpliendo con el perfil propuesto por la carrera y los estudiantes adquiriendo capacidades para su empleabilidad, no son los únicos beneficiados con la inclusión de las prácticas profesionales en el currículo. Las organizaciones que dan acceso a los profesionistas en formación reciben de ellos sus conocimientos, tiempo y habilidades; estos contribuyen al desarrollo y funcionamiento organizacional sin establecer una relación laboral (p. 29). 
Los cuestionamientos acerca de la formación profesional son compartidos a nivel global, la IFAC (Federación Internacional de Contadores) también ha emitido una serie de pronunciamientos mediante los cuales "establece los requerimientos sobre la formación del contador público, la evaluación de sus capacidades y competencias, así como los requisitos de práctica previa para obtener la matriculación”.

Esta investigación bibliográfica busca describir los diferentes enfoques presentados por teóricos y los resultados de investigaciones sobre la incidencia de las pasantías en el perfil del profesional contable.

La revisión se realizó, partiendo de la hipótesis de que las pasantías profesionales inciden en el logro del perfil del profesional contable; entiéndase que, si bien las IES proponen las mismas como requisito en los proyectos para habilitación de carreras, esto es más profundo y fundamentado en el logro de perfiles técnicos que redundará con beneficios en el mercado laboral y comercial. Con el análisis de las apreciaciones y resultados de investigaciones, se realizó la contrastación de las mismas que facilitaron la realización de las conclusiones.

Para llegar al objetivo propuesto, el trabajo se estructura de la siguiente forma, contiene una introducción con la descripción del propósito del estudio, la problemática que propicia el análisis y el estado del arte logrado mediante la revisión bibliográfica. Posteriormente se explica la metodología adoptada y la forma de acceso a los estudios científicos existentes en el tema. Se concluye con el resultado, discusión y conclusiones a las cuales se lograron llegar.

El tema de las pasantías profesionales desde hace unos años ha generado varios espacios de debate considerando la pertinencia de las actividades que desempeñan en los diferentes ámbitos o espacios de práctica como así también con respecto a la contribución de las mismas en la formación de capacidades relacionadas con el perfil propuesto por la carrera. Con respecto al tema Camilloni, (2013) plantea que las prácticas profesionales son necesarias, al igual que cualquier otra actividad académica para el entrenamiento profesional, requieren que sean planificadas y supervisadas cuidadosamente, para así garantizar su funcionamiento y promover el aumento de la calidad.

Los procesos de formación bien pueden presentar debilidades, no obstante, identificarla y tratar de subsanarlas es la mejor opción; la Organización Internacional del Trabajo, respecto al tema de las pasantías, refiere que con frecuencia son consideradas una 
excelente manera para adquirir experiencia laboral y afianzarse en el mercado de trabajo, pero que, paralelamente, son un riesgo latente para los jóvenes, ya que se han presentado numerosas denuncias de abusos, pues éstas son consideradas como fuente de trabajo a bajo costo o, incluso, gratuito, reconociendo que esta garantía para la juventud puede ser muy eficaz para reducir el desempleo y el desaliento en el que se encuentra, destacando que paralelamente se necesita supervisión y evaluación de su impacto. (Rueda Rodríguez, 2014, p. 127).

Otro tema son los requerimientos de los empleadores, si bien los alumnos que tiene la oportunidad de acceder a estos espacios laborales, deberían desempeñar funciones relacionadas a su carrera, las interrogantes son: ¿alguien evalúa su desempeño? ¿Cada cuanto los evalúan? ¿qué tipo de evaluaciones realizan? ¿las evaluaciones evidencian las capacidades obtenidas por los estudiantes en esas pasantías? y, por último ¿se logró llegar al perfil propuesto por la carrera? ¿se realiza un análisis sobre la incidencia de las mismas en el logro del perfil propuesto?

Sobre el mismo planteamiento, Camilloni, (2013) señala que, las prácticas profesionales se enfocan en el aprendizaje de conocimientos específicos de la profesión y no en el servicio a la comunidad o a la formación de la ciudadanía, tal como lo hacen el servicio social u otras actividades.

Siguiendo el mismo enfoque de lo planteado en el párrafo anterior, Rodríguez y Seda (2013) retoman el planteamiento de Fortes y Lominitz (1991) afirman que el desarrollo profesional se ve beneficiado con la inclusión de actividades prácticas ya que por medio de estas el alumno tiene oportunidad de observar y ejecutar las funciones, normas, valores y acciones de su profesión, los estudiantes tienen la posibilidad de demostrar su capacidad de actuación y adaptación al trabajo real, lo que se traduce en una puerta de entrada para el establecimiento de futuras relaciones laborales.

Por su parte, Cueva Navas et al., (2014) sostienen que muchos de los programas en la educación superior usan sistemas de prácticas que ubican a los estudiantes, exclusivamente, en un contexto de trabajo (ellos no toman clases teóricas simultáneamente) por un extenso período de tiempo. El período de tiempo puede variar con base en la naturaleza de la pasantía, los requerimientos académicos de los estudiantes, al igual que los requisitos de los programas y pueden cambiar por algunas semanas al año. Aparte de los obvios beneficios del estudiante, al estar en la capacidad de enfocarse, 
exclusivamente, en el desarrollo de su trabajo, este sistema es relativamente fácil de administrar (p. 137).

De Ibarrola (2002), destaca sobre el conocimiento, como el factor que constituye una fuerza productiva de primera importancia, se redefine y evoluciona la noción del mismo y ya no se distingue entre teoría y práctica o conocimiento abstracto y aplicado, sino que en el desempeño laboral lo que importa es la integración de ambos para sacar adelante las tareas de producción y resolver problemas con base en el conocimiento. Si tomamos en cuenta este relevante marco de referencia podríamos decir que las distinciones tajantes entre estos dos conceptos parecen obsoletas. Las exigencias de la formación profesional apuntan a la necesidad de integrar la teoría y la práctica para que se genere el conocimiento general y, por ende, también aquel que tiene valor para el ejercicio de una profesión que responde a las necesidades del mercado laboral.

Lo mencionado con antelación se torna complicada, porque si bien este tipo de mecanismo ofrece una serie de ventajas para los jóvenes otorgándoles conocimientos propuestos en el perfil de egreso, existe el peligro latente de que las referidas prácticas, al no contar con una organización adecuada y específica en la que se establezca claramente los procedimientos y objetivos conllevará a fuertes críticas del proceso de transición escuela-trabajo y que en realidad los jóvenes terminen estancándose sin conseguir conocimientos nuevos que los preparen para un futuro laboral exitoso.

\section{Pasantía conceptualización e importancia}

En relación a las pasantías según la Real Academia de la Lengua (2013) es: “el ejercicio del pasante en las facultades y profesiones que asiste y acompaña al maestro de una facultad en el ejercicio de ella, para imponerse enteramente en su práctica”.

Quispe et al., (2014) también realizan una apreciación sobre el mismo tema, afirma que, a pesar de la trascendencia de las prácticas profesionales en la formación de los estudiantes de educación superior, dada a su función como vínculo entre las IES y la sociedad, estas suelen ser nombradas de diversas formas: practicum, estancia profesional, estancia universitaria, entre otras. La variación puede depender de la institución, la zona o la disciplina.

Sobre su importancia, Rama (2011) señala que las mismas contribuyen a viabilizar la pertinencia de los aprendizajes y facilitan además la empleabilidad en tanto permiten la adquisición de las habilidades y experiencias que requiere el ingreso al mundo del trabajo. 
Finalmente, las pasantías estudiantiles a nivel del grado o las prácticas profesionales a nivel del postgrado se constituyen una de las formas que está asumiendo el enfoque curricular por competencias, orientado a facilitar el pasaje del "saber" hacia el "saber hacer" (p. 33). Haciendo referencia específicamente a los objetivos Alvarez (2003) en su libro Manual de apoyo para la implementación de Pasantías Estudiantiles menciona que "El objetivo principal es la vinculación educación - trabajo" (pp.11, 12).

También Vega Villacís et al., (2020) hacen referencia a que las prácticas y/o pasantías pre-profesionales podrán realizarse a lo largo de la formación de la carrera de forma continua o no y deberán ser coherentes con los resultados de aprendizaje y el perfil de la carrera. Son actividades de aprendizaje orientadas a la aplicación de conocimientos y al desarrollo de competencias profesionales que se planifican mediante planes, programas y/o proyectos (p. 939).

\section{Beneficiarios de las pasantías}

Es relevante señalar que las IES y los estudiantes son claramente beneficiados con las pasantías; Gutiérrez Bernal et al., (2019) mencionan que las IES cumpliendo con el perfil propuesto por la carrera y los estudiantes adquiriendo capacidades para su empleabilidad, sin embargo, no son los únicos beneficiados con la inclusión de las prácticas profesionales en el currículo. Las organizaciones que dan acceso a los profesionistas en formación reciben de ellos sus conocimientos, tiempo y habilidades; estos contribuyen al desarrollo y funcionamiento organizacional sin establecer una relación laboral (p. 29).

Gutiérrez Bernal et al., (2019) enfatizan, las prácticas profesionales son el espacio ideal para que las IES identifiquen cuáles son las demandas de la sociedad y el campo laboral. Gracias a ellas, las IES pueden incluir en sus currículos las asignaturas y estrategias de enseñanza-aprendizaje apropiadas que contribuyan a la mejor formación de sus egresados; es decir, son un punto de apoyo para su evaluación y la toma de decisiones para la mejora de todo el sistema (p. 29).

Para lograr esta vinculación indudablemente el egresado debe adquirir ciertas capacidades, sobre las mismas y su conceptualización, Gimeno Sancristán (2001) menciona, la competencia se enuncia en el sistema educativo en la nueva modernidad, espacio y tiempo en el cual se redefinen las jerarquías de valores, para Mertens (1996) la competencia laboral consiste en la aptitud de un individuo para desempeñar una misma 
función productiva en diferentes contextos y con base en los requerimientos de calidad esperados por el sector productivo. Esta aptitud se logra con la adquisición y desarrollo de conocimientos, habilidades y capacidades que son expresados en el saber, el hacer y el saber hacer (p. 119).

\section{Perfil profesional contable requerimientos de formación}

Con respecto al perfil profesional contable, se halla el Proyecto Tuning para América Latina 2004 - 2006 que surge en un contexto de intensa reflexión sobre Educación Superior tanto a nivel regional como internacional, propone cuatro grandes líneas a saber: las competencias de egreso (genéricas y específicas), enfoque de enseñanza, aprendizaje y evaluación, créditos académicos y calidad de programas; se plantean nuevos esquemas a partir de sus estudios, dándose un cambio radical en la educación superior, donde se resalta la diversidad en fuentes y formas de aprendizaje, la orientación al auto aprendizaje, la promoción de las capacidades de autogeneración de trabajo, de interacción y de trabajo en equipo, el requerimiento de un idioma extranjero y la formación general (no profesionalizante) (Universidad de Deusto / Universidad de Groningen, 2007).

Desde la perspectiva del modelo de formación profesional de la Federación Internacional de Contadores (IFAC) ante las dudas y cuestionamientos acerca de la formación profesional han emitido una serie de pronunciamientos mediante los cuales "establece los requerimientos sobre la formación del contador público, la evaluación de sus capacidades y competencias, así como los requisitos de práctica previa para obtener la matriculación" De ellos, los más importantes o significativos son las denominadas "Internacional Education Standards" - IES, es decir, normas internacionales de educación, que a la fecha son las siguientes:

IES 1 - Requisitos de ingreso a un programa de educación contable profesional.

IES 2 - Contenido de los programas de educación profesional contable.

IES 3 - Habilidades profesionales y educación general.

IES 4 - Valores profesionales, ética y actitudes.

IES 5 - Requisitos de experiencia práctica.

IES 6 - Evaluación de las capacidades y de las competencias profesionales.

IES 7 - Desarrollo profesional continuo.

IES 8 - Competencias requeridas para auditores profesionales. (IFAC (Federación Internacional de Contadores), s.f.) 
Drucker (1993) dice que "El saber es hoy el único recurso significativo. Los tradicionales factores de producción, suelo (recursos naturales), mano de obra y capital, no han desparecido, pero se han convertido en secundarios; pueden obtenerse, y con facilidad, siempre que haya saber; y el saber en su nuevo significado es saber en tanto que servicio..." También Weinberg (1996) manifiesta, "las concepciones clásicas de formación la conciben como la transmisión ordenada y sistemática de un conjunto de conocimientos, habilidades y destrezas que le permiten al trabajador una elevación de sus calificaciones personales, pero descontextualizada del entorno y el tiempo en que se lleva a cabo y no necesariamente articulada con los procesos de trabajo para los que está contemplada". El nuevo enfoque de formación para las competencias se basa en que "las competencias modernas no se enseñan en un curso, sino que son el reflejo de un ambiente productivo, empapado en la atmósfera de las empresas, en los códigos de conducta y funcionamiento que operan en la realidad".

\section{ESTRATEGIAS METODOLÓGICAS o MATERIALES Y MÉTODOS}

Para la realización de esta investigación bibliográfica se procedió a la revisión de artículos de diversos autores sobre el tema accediendo a ellos por medio de Google Académico y por el portal Cicco de Conacyt Paraguay, se realizó la búsqueda utilizándose las palabras clave: pasantía profesional y perfil del profesional contable. Se efectuó la revisión de algunas bibliografías de los años 2003, 2008 y 2010, posteriormente se dio énfasis en la búsqueda de materiales de los años 2016 hasta 2020, consecutivamente se procedió a la elaboración de fichas bibliográficas, en las mismas se asentaron datos de los materiales, año, título y objetivo del trabajo; se realizó el análisis exhaustivo de textos para incorporarlos a este trabajo considerando las fuentes, referencias de las mismas y las Normas de Estilo Apa.

Los criterios de inclusión considerados para la elección de los artículos estaban relacionados al logro del objetivo de esta investigación ya que existe mucha información y diferentes perspectivas sobre las pasantías profesionales y el perfil profesional del profesional contable; los años de publicación de los artículos y la confiabilidad de fuentes (artículos científicos publicadas por revistas indexadas y algunos trabajos de grado).

\section{RESULTADOS Y DISCUSIÓN}

Mediante esta investigación se lograron recabar los siguientes aportes teóricos; con respecto a las pasantías tanto Rama (2011) como Mertens (1996) señalan que las pasantías 
o las prácticas profesionales a nivel del postgrado se constituyen una de las formas que está asumiendo el enfoque curricular por competencias, orientado a facilitar el pasaje del "saber" hacia el "saber hacer, consiste en la aptitud de un indivíduo para desempeñar una misma función productiva en diferentes contextos y con base en los requerimientos de calidad esperados por el sector productivo. Esta aptitud se logra con la adquisición y desarrollo de conocimientos, habilidades y capacidades que son expresados en el saber, el hacer y el saber hacer.

Desde la perspectiva de Vega Villacís et al., (2020) las prácticas y/o pasantías preprofesionales podrán realizarse a lo largo de la formación de la carrera de forma continua o no y deberán ser coherentes con los resultados de aprendizaje y el perfil de la carrera. Este proceso según Gutiérrez Bernal et al., (2019) se concreta mencionando a las IES quienes cumpliendo con el perfil propuesto por la carrera y los estudiantes adquiriendo capacidades para su empleabilidad se podría lograr que cumplan su objetivo professional. Se puede observar en estas apreciaciones que la prioridad es que por medio de las pasantías el alumno adquiera y desarrollo conocimientos, habilidades y capacidades; es interesante analizarlo desde el punto de vista del mercado empresarial, asumiendo que los alumnos apuntan a desenvolverse laboralmente en el mismo y en un mercado laboral donde cada día es más relevante destacarse profesionalmente por lo tanto las pasantías deben contribuir para ello; como segundo punto se menciona la intervención de la IES, de ellas se plantea la necesidad de que las actividades de pasantías se enfoquen a obtener resultados de aprendizaje con el perfil propuesto por la misma.

Haciendo alusión al perfil del profesional contable, se halla propuesto específicamente mediante un estudio realizado durante el Proyecto Tuning para América Latina 2004 2006 que surge en un contexto de intensa reflexión sobre Educación Superior tanto a nivel regional como internacional, se propone cuatro grandes líneas a saber: las competencias de egreso (genéricas y específicas), enfoque de enseñanza, aprendizaje y evaluación, créditos académicos y calidad de programa; se resalta la diversidad en fuentes y formas de aprendizaje, la orientación al auto aprendizaje, la promoción de las capacidades de autogeneración de trabajo, de interacción y de trabajo en equipo, el requerimiento de un idioma extranjero y la formación general.

Otra propuesta surge desde la perspectiva del modelo de formación profesional de la Federación Internacional de Contadores (IFAC), es relevante mencionar los puntos 
específicos: IES 5 - Requisitos de experiencia práctica; IES 6 - Evaluación de las capacidades y de las competencias profesionales; IES 7 - Desarrollo profesional continuo; IES 8 - Competencias requeridas para auditores profesionales.

Desde ambas perspectivas se menciona la importancia de adquirir las competencias necesarias para el profesional incluyendo la experiencia práctica, capacidades y competencias profesionales requeridas para su desempeño profesional; para estas recomendaciones se puede asumir la propuesta más viable es la realización de pasantías o prácticas profesionales.

También Weinberg (1996) manifiesta que el nuevo enfoque de formación para las competencias se basa en que "las competencias modernas no se enseñan en un curso, sino que son el reflejo de un ambiente productivo, empapado en la atmósfera de las empresas, en los códigos de conducta y funcionamiento que operan en la realidad".

\section{CONCLUSIÓN O CONSIDERACIONES FINALES}

El trabajo se sustenta en la problemática de las pasantías y su incidencia en el perfil del profesional contable, tema que ha originado debates considerando específicamente la pertinencia de las actividades que desempeñan en los diferentes ámbitos o espacios de práctica como así también con respecto a la contribución de las mismas en la formación de capacidades relacionadas con el perfil propuesto por la carrera.

Los hallazgos teóricos refieren que las pasantías con frecuencia son consideradas una excelente manera para adquirir experiencia laboral y afianzarse en el mercado de trabajo, pero que, paralelamente, son un riesgo latente para los jóvenes destacando que necesita supervisión y evaluación de su impacto.

Las distintas apreciaciones hacen referencia a que por medio de las pasantías el alumno puede adquirir conocimientos, habilidades y capacidades que son expresados en el saber, el hacer y el saber hacer; estas capacidades en el ámbito laboral son esenciales considerando un mercado laboral donde la productividad de los empleados es el evaluado como relevante y excluyente inclusive; es aquí donde se incluye el papel de las IES para la ejecución eficaz de estas actividades académicas.

En relación al perfil del profesional contable, los referentes de este campo profesional sostienen que estos profesionales deben adquirir conocimientos mediante experiencia práctica, hacen énfasis en la necesidad asumir un nuevo enfoque de formación para las 
competencias y que los alumnos se relacionen a las empresas, con los códigos de conducta y funcionamiento que operan en la realidad.

Con estos hallazgos bibliográficos se puede inferir que efectivamente las pasantías inciden en el logro del perfil propuesto por las IES, sin embargo, para futuras investigaciones sería de gran importancia comprobar mediante mediciones y aplicación de instrumentos para recolección de datos en trabajo de campo, el nivel o grado de incidencia de muestras específicas.

\section{LISTA DE REFERENCIAS}

Alvarez, A. (2003). Manual de apoyo para la implementación de pasantías educativas. Buenos Aires, Argentina: Artes Gráficas Integradas.

Camilloni, A. (2013). La inclusión de la educación experiencial en el currículo universitario. Integración docencia y extensión: otra forma de enseñar y de aprender. Universidad Nacional del Litoral, 11-21.

Castillo, J., Álvarez, N., \& Torres, A. (2013). La vinculación de las universidades mexicanas con el entorno: expresión de responsabilidad social. Transformación, 9(2), 1-13.

Cueva Navas, A., Fernández Machado, L., López Molina, D., \& Van Hoof, H. (enero de 2014). Percepciones de los Estudiantes sobre las Prácticas como Parte del Programa de Estudios de Hospitalidad: Un estudio cualitativo. Publicación Semestral(26). México: El Periplo Sustentable. Universidad Autónoma del Estado de México. Obtenido de www.psus.uaemex.mx

De Ibarrola, M. (27 de junio de 2002). Nuevas tendencias de la formación profesional para el trabajo. Ponencia presentada en el encuentro Desarrollo regional, integración continental y formación de los jóvenes para el trabajo. México, México.

Drucker, P. (1993). La sociedad poscapitalista. Buenos Aires: Ed. Sudamericana.

Gimeno, S. J. (2006). La reforma necesaria entre la política educativa y la práctica escolar. Madrid, España: Ediciones Morata.

Gutiérrez Bernal, Z. G., Farfán García, M. d., \& Navarrete Sánchez, E. (Julio-Diciembre de 2019). Evaluar las prácticas profesionales: una posibilidad de mejora para la educación superior en México. PSICUMEX, Vol. 9(No. 2), 22-34. 
Ibarrola, M. (1986). La Educación Superior en México. Caracas, Venezuela: CRESALCUNESCO.

IFAC (Federación Internacional de Contadores). (s.f.). Obtenido de https://www.ccpy.org.py/ifac

Mertens, L. (1996). Competencia laboral: sistemas, surgimientos y modelos. Montevideo: Cinterfor.

Quispe, A., Victorino, L., \& Atriano, R. (2014). Vinculación de Instituciones de Instituciones de Educación Agrícola Superior (IEAS) con sectores productivos. El caso de la residencia profesional en el Instituto Tecnológico del Altiplano de Tlaxca (ITAT). Revista de la Educación Superior, 43(170), 135-152. Obtenido de http://www.redalyc.org/articulo.oa?id=60431363007

Rama, V. (enero-marzo de 2011). La incorporación de la práctica estudiantil en las universidades latinoamericanas como nuevo paradigma educativo. Revista Universidades, vol. LXI(núm. 48), 33- 45.

Real Academia de la Lengua. (2013). Obtenido de www.rae.es

Rodríguez, F., \& Seda, I. (2013). El papel de la participación de estudiantes de Psicología en escenarios de práctica en el desarrollo de su identidad profesional. Perfiles educativos, 35(140), 82-99. Obtenido de http://www.redalyc.org/articulo.oa?id=1322615600

Rueda Rodríguez, A. E. (julio-diciembre de 2014). Las prácticas profesionales y las pasantías desde la legislación comparada. Revista Latinoamericana de Derecho Social(Núm. 19), 111-132. Obtenido de http://www.scielo.org.mx/pdf/rlds/n19/1870-4670-rlds-19-00111.pdf

Silva Laya, M. (2008). ¿Contribuye la universidad tecnológica a formar las competencias necesarias para el desempeño profesional?. Un estudio de caso.

Revista Mexicana de Investigación Educativa, 13(38), 773-800. Obtenido de https://www.redalyc.org/articulo.oa?id=14003805

Universidad de Deusto / Universidad de Groningen. (2007). Tuning America Latina. Reflexiones y perspectivas de la Eduación Superior en América Latina. Bilbao: Universidad de Deusto. Obtenido de http://tuningacademy.org/wpcontent/uploads/2014/02/TuningLAIII_Final-Report_SP.pdf 
Vega Villacís, G., Alcoser Cantuña, F., Guevara Albán, G., \& Guevara Albán, C. (2020). Estrategias metodológicas en instituciones de educación superior para el desarrollo de prácticas y/o pasantías preprofesionales en estado de excepción y distanciamiento social por emergencia COVID-19, caso Universidad Técnica de Babahoyo. Journal of Science and Research, Vol. 5(Núm. CIINGEC2020).

Weinberg, P. D. (1996). La construcción de una nueva institucionalidad para la formación. Documento presentado en el Seminario Educación, Tecnología y Empleo. Mendoza. 